BULL. AUSTRAL. MATH. SOC.

VOL. 20 (1979), 199-206.

\title{
Interchange of vector valued integrals when the measures are Bochner or Pettis indefinite integrals
}

\section{Andre de Korvin and Charles E Roberts , Jr}

\begin{abstract}
Necessary and sufficient conditions for the interchange of two Bochner integrals and for the interchange of two Pettis integrals are obtained. These conditions are different from those generally required in classical Fubini theorems since they do not require the construction of the cross product measure. The proof makes use of the Vitali-Hahn-Saks Theorem. It should be noted that while Fubini theorems use the cross product measure, one of the difficulties encountered is that the product measure fails to be countable additive - this is pointed out in $M$. Bhaskara Rao (Indiana Univ. Math. J. 21 (1972), 847-848) and Charles Swartz (Bull. Austral. Math. Soc. 8 (1973), 359-366). Most applications require the interchange of the two integrals rather than integration with respect to the product measure.
\end{abstract}

In recent years much work has been done in generalizing Fubini's Theorem. Articles [1], [2], [3], [8], [11], [12] are a small sample of such work. In [1] it is shown that

$$
\int_{H} d K_{2} \int_{H} d K_{1}[f \cdot x]=\int_{H \times H} d\left(K_{1} \times K_{2}\right)[f \cdot x],
$$

where $H$ is a compact space and where $K_{1}$ has values in $B\left(X, Y^{* *}\right)$ and $K_{2}$ has values in $B\left(Y^{* *}, Z^{* *}\right)$. A similar result is shown in [2] where

Received 30 January 1979. 
$f \cdot x$ is replaced by a function $f$ from $H \times T$ into $E$ which is integrable with respect to $v\left(K_{1}\right) \times v\left(K_{2}\right)$ where $v(m)$ represents the variation of the vector valued measure $m$. Results of [8] show that if $\mu$ and $\nu$ are regular vector valued measures defined on Borel sets then $\mu \times \nu$ is well defined and the Fubini Theorem holds provided a separability condition is assumed on $v$. In [11] it is assumed that $\mu$ and $v$ are bounded and finitely additive and that $f$ is a real valued function which is bounded. Under certain assumptions it is shown that $\iint f d \mu d v=\iint f d v d \mu$. Finally in [12] the following is shown. Let $M$ and $N$ denote two $\sigma$-algebras of subsets of a set $S$ and $T$ respectively. Let $X$ and $Y$ be two Banach spaces and let $($,$) denote a continuous$ bilinear map of $X \times Y$ into the scalars. Let $\lambda$ be a set function defined on rectangles $A \times B$ where $A \in M$ and $B \in N$, by

$$
\lambda(A \times B)=\langle m(A), n(B)\rangle,
$$

where $m$ is a vector measure from $M$ into $X$ and $n$ is a vector measure from $N$ into $Y$. Then under certain conditions

$$
\begin{aligned}
\int_{T}\left\langle\int_{S} H(s, t) d m(s), d n(t)\right\rangle & =\int_{S}\left\langle\int_{T} H(s, t) d n(t), d n(s)\right\rangle \\
& =\iint_{S \times T} H(s, t) d \lambda(s, t),
\end{aligned}
$$

where $H$ is scalar valued and satisfies some conditions and the first two integrals are in the sense of [5].

It is important to note that in general $\lambda$ as defined above does not have a countable additive extension to the $\sigma$-algebra generated by rectangles. In [10] an example illustrating this is constructed. In [12] Swartz considers the case in which $m$ and $n$ are of the form

$$
m(E)=\int_{E} f d \alpha \text { and } n(F)=\int_{F} g d \beta
$$

where $f$ (and $g$ ) are strongly measurable $X$ (and $Y$ ) valued and Pettis integrable with respect to the o-finite positive measure $\alpha$ (and $B$ ). The reader is referred to [9] for basic properties of the Pettis integral. Let

$$
P(s, t)=\langle f(s), g(t)\rangle
$$


It is shown that if $P$ is $\alpha \times \beta$ integrable, then $\lambda$ can be extended to a countably additive set function over the $\sigma$-algebra generated by rectangles. In this case the Fubini Theorem as stated above holds for $H: S \times T \rightarrow R$ where $H$ is bounded and scalar valued. Furthermore, if $f$ and $g$ are Bochner integrable rather than Pettis integrable, the requirement that $H$ be bounded may be replaced by the requirement that $H$ be integrable with respect to $v(m) \times v(n)$.

The main purpose of the present article is to provide a necessary and sufficient condition for

$$
\begin{aligned}
\int_{T}\left\langle X_{F}(t) \int_{S} X_{E}(s) H(s, t) d m(s), d n(t)\right\rangle & \\
& =\int_{S}\left\langle X_{E}(s) \int_{T} X_{E}(t) H(s, t) \operatorname{dn}(t), d m(s)\right\rangle
\end{aligned}
$$

where $E \in M, F \in N$, and where $\mathrm{X}_{A}$ denotes the characteristic function of $A$. Of course if $f$ and $g$ are Pettis integrable and $H$ is bounded or if $f$ and $g$ are Bochner integrable and $H$ is $v(m) \times v(n)$ integrable, the above equality is true by [12]. It should be stressed that we do not assume these conditions on $H$. Our first result pertains to the case in which $f$ and $g$ are Bochner integrable and our second result assumes that $f$ and $g$ are Pettis integrable. For elementary properties of such integrals see [5], [6], [7], and [9]. Finally the reader is referred to [4] for comparative properties of the Bochner, Pettis, and Gelfand-Pettis integral.

THEOREM 1. Assume $P$ is integrable with respect to $\alpha \times \beta$ and that $m$ (and $n$ ) are Bochner indefinite integrals of $\alpha$ (and $\beta$ ); then

$$
\begin{aligned}
\int_{T}\left\langle X_{F}(t) \int_{S} X_{E}(s) H(s, t) d m(s), d n(t)\right\rangle & \\
& =\int_{S}\left\langle X_{E}(s) \int_{T} X_{F}(t) H(s, t) d n(t), d m(s)\right\rangle
\end{aligned}
$$

for all $E \in M$ and $E \in N$ if, and only if,

(1) $\int H(s, t) d m(s)$ is integrable with respect to $n$, and

(2) $H(s, t)$ is integrable with respect to $n$ (m almost everywhere). 
Proof. If the above equality holds it is quite clear that by making $E=S$ and $E=T$, (1) and (2) hold.

We now assume (1) and (2). From (1) it follows that

$$
\left\|\int_{S} H(s, t) d m(s)\right\|<\infty, n \text { almost everywhere. }
$$

Thus

$$
\int|H(s, t)| d v(m)(s)<\infty, n \text { almost everywhere. }
$$

For every positive integer $k$ let

$$
T(k)=\left\{t \in T\left|\int\right| H(s, t) \mid d v(m)(s) \leq k, n \text { almost everywhere }\right\} .
$$

Then $T(k) \uparrow T$ almost everywhere $(n)$ and $v(n)[T(k)]<\infty$ since $f$ and $g$ Bochner integrable implies that $m$ and $n$ have finite variation. Now

$$
\chi_{F \cap \mathbb{T}(k)} X_{E}|H(s, t)|
$$

is integrable with respect to $v(m) \times v(n)$, since

$$
\begin{aligned}
\iint X_{F \cap T(k)}(t) X_{E}(s) \mid H(s, t) & \mid d[v(m) \times v(n)](s, t) \\
= & \int_{T} X_{F \cap T(k)}(t)\left[\int_{S} X_{E}(s)|H(s, t)| d v(m)(s)\right] d v(n)(t) \\
\leq & \int_{T} k \chi_{F \cap T(k)}(t) d v(n)(t) \leq k v(n)[T(k)]<\infty .
\end{aligned}
$$

The above steps are justified by Tonelli's Theorem - see [6]. Now by [12] it follows that

$$
\begin{aligned}
\int_{T}\left\langle X_{E \cap T(k)}(t) \int_{S} X_{E}(s) H(s,\right. & t) d m(s), d n(t)\rangle \\
& =\int_{S}\left\langle d m(s), X_{E}(s) \int_{T} X_{F \cap T(k)}(t) H(s, t) d n(t)\right\rangle \\
& =\iint_{S \times T} X_{F \cap T(k)}(t) X_{E}(s) H(s, t) d \lambda(s, t)=h_{k}(E) .
\end{aligned}
$$

Clearly $h_{k}$ are scalar measures defined on $M$. Also $h_{k} \ll \operatorname{var}(m)$. Let

$$
f_{k}(t)=\chi_{F \cap T(k)}(t) \int_{S} \chi_{E}(s) H(s, t) d n(s)
$$


Then $h_{k}(E)=\int_{T}\left\langle f_{k}, d n\right\rangle$. Now the sequence $\left\{f_{k}\right\}$ converges $n$ almost everywhere to

$$
\chi_{E} \int_{S} X_{E}(s) H(s, t) d m(s)
$$

for each $f_{k}$ is an n-integrable function from $T$ into $X$. Also

$$
\left\|f_{k}\right\|_{X} \leq\left\|\int_{S} \chi_{E}(s) H(s, t) d m(s)\right\|_{X} .
$$

Thus each $f_{k}$ is dominated by a function that is Bochner integrable with respect to $n$. Thus

$$
\chi_{E}(t) \int_{S} \chi_{E}(s) H(s, t) d m(s)
$$

is Bochner integrable with respect to $n$ and $h_{k}(E)$ converges to

$$
\int_{T}\left\langle X_{E}(t) \int_{S} X_{E}(s) H(s, t) d m(s), d n(t)\right\rangle,
$$

which we denote by $h(E)$.

Now let

$$
g_{k}(s)=\int_{T} \chi_{F \cap T(k)}(t) H(s, t) d n(t)
$$

Then all $g_{k}$ are functions from $S$ into $Y$ and

$$
h_{k}(E)=\int_{E}\left\langle\operatorname{dm}(s), g_{k}(s)\right\rangle .
$$

Since

$$
v\left(h_{k}\right)(E)=\int_{E}\left\|g_{k}(s)\right\|_{Y} d v(m)(s),
$$

it follows that $v\left(h_{k}\right) \ll v(m)$. By the Vitali-Hahn-Saks Theorem [6, p. 150] it follows by (2) and the dominated convergence theorem that since $g_{k}(s)$ converges $m$ almost everywhere to

$$
\int_{T} \chi_{F}(t) H(s, t) d n(t)
$$


it converges in the $L^{1}$ norm to that function. Indeed since $h_{k}(E)$ converges to $h(E)$ by the Vitali-Hahn-Saks Theorem the $h_{k}$ are uniformly absolutely continuous with respect to $v(m)$, and since $h_{k}$ is scalar valued it follows that $v\left(h_{k}\right)$ are uniformly absolutely continuous with respect to $v(m)$. (Note that since $f$ is Bochner integrable, $v(m)(s)<\infty$.) Thus

$$
\begin{aligned}
\lim h_{k}(E) & =\int_{S}\left\langle d m(s), X_{E}(s) \int_{T} x_{F}(t) H(s, t) d n(t)\right\rangle \\
& =\int_{T}\left\langle x_{F}(t) \int_{S} x_{E}(s) H(s, t) d m(s), d n(t)\right\rangle .
\end{aligned}
$$

This finishes the proof of the theorem.

We now assume that $f$ and $g$ are Pettis-integrable. Of course it is not true anymore that $v(m)$ and $v(n)$ are finite. It is shown in [12] that in this case the Fubini Theorem holds provided $H$ is bounded. It is also noted that while the restriction that $H$ be bounded is undesirable, it is difficult to give conditions that insure that $\int H(s, t) f(s) d \alpha(s)$ exists as a Pettis-integral.

THEOREM 2. Assume $P$ is $\alpha \times \beta$ integrable, that $m$ and $n$ are Pettis indefinite integrals and that a sequence $\left\{F_{j}\right\}$ may be found in $N$ such that $F_{j}+T, n$ almost everywhere, $\chi_{F_{j}}(t) H(s, t)$ is bounded, and $v(n)\left(F_{j}\right)<\infty$. Then the conclusion of the previous theorem holds.

Proof. Again the integrals with respect to $m$ and $n$ are taken in the sense of [5] or [6]. For each positive integer $k$ define

$$
T(k)=\left\{t \in \bigcup_{j=1}^{k} F_{j}\left|\int\right| H(s, t) \mid d v(m)(s) \leq k\right\} .
$$

Again $T(k)+T, n$ almost everywhere, and $v(n)[T(k)]<\infty$. The function

$$
X_{E \cap T(k)}(t) X_{E}(s)|H(s, t)|
$$

is bounded. Thus by [12] we obtain $\left\{h_{k}\right\}$ as in Theorem 1 such that $h_{k}$ 
are finite measures. Again the use of the Vitali-Hahn-Saks Theorem completes the proof. Since $v(m)$ is $\sigma$-finite we can find $E_{\varepsilon} \in M$ such that $v\left(h_{k}\right)\left(S-E_{\varepsilon}\right)<\varepsilon$ with $v(m)\left(E_{\varepsilon}\right)<\infty$. The rest of the proof follows as in Theorem 1 .

\section{References}

[1] Richard A. Alò and Andre de Korvin, "A one-sided Fubini theorem for Gowurin measures", J. Math. Anal. App Z. 38 (1972), 387-398.

[2] U.K. Bandyopadhyay, "On products of vector measures", J. Austral. Math. Soc. Ser. A 19 (1975), 91-96.

[3] Marc A. Berger and Victor J. Knight, "A Fubini theorem for iterated stochastic integrals", Bull. Amer. Math. Soc. 84 (1978), $159-160$.

[4] J. Diestel, "Applications of weak compactness and bases to vector measures and vectorial integration", Rev. Roumaine Math. Pures App Z. 18 (1973), 211-224.

[5] N. Dinculeanu, Vector measures (Pergamon Press, Oxford, London, Edinburgh, New York, Toronto, Sydney, Paris, Braunschweig, 1967).

[6] Nelson Dunford and Jacob J. Schwartz, Linear operators, Part I (Interscience [John Wiley \& Sons], New York, London, 1958).

[7] I. Gelfand, "Abstrakte Funktionen und lineare Operatoren", Rec. Math. Moscou (2) 4 (1938), 235-286.

[8] James E. Huneycutt, Jr., "Products and convolutions of vector valued set functions", Studia Math. 41 (1972), 119-129.

[9] B.J. Pettis, "On integration in vector spaces", Trans. Amer. Math. Soc. 44 (1938), 277-304.

[10] M. Bhaskara Rao, "Countable additivity of a set function induced by two vector-valued measures", Indiana Univ. Math. J. 21 (1972), 847-848. 
[11] George Edward Sinclair, "A finitely additive generalization of the Fichtenholz-Lichtenstein theorem", Trans. Amer. Math. Soc. 193 (1974), 359-374.

[12] Charles Swartz, "The product of vector-valued measures", Bulz. Austral. Math. Soc. 8 (1973), 359-366.

Department of Mathematics,

Indiana State University,

Terre Haute,

Indiana,

USA. 